\title{
Status of correlation between BMI and response to immunocheck-point inhibitor in advanced non-small-cell lung cancer
}

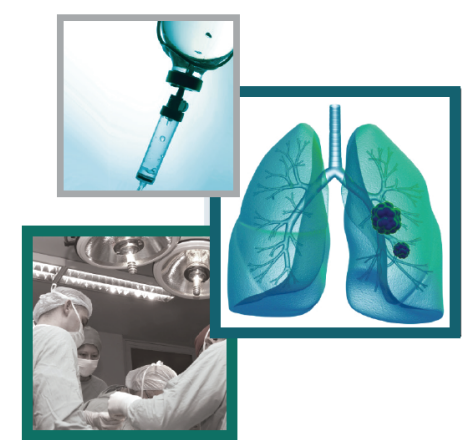

\author{
Alain Gelibter ${ }^{*, 1}$, Mario Occhipinti ${ }^{1}$ (D) Simona Pisegna ${ }^{1}$, Alessio Cortellini ${ }^{2,3}$, Enrico \\ Cortesi ${ }^{1}$ \& Paolo Marchetti ${ }^{1,4}$ \\ ${ }^{1}$ Medical Oncology Unit, Policlinico Umberto I, Sapienza University of Rome, Rome, Italy \\ ${ }^{2}$ Medical Oncology Unit, St. Salvatore Hospital, L'Aquila, Italy \\ ${ }^{3}$ Department of Biotechnological \& Applied Clinical Sciences, University of L'Aquila, L'Aquila, Italy \\ ${ }^{4}$ U.O.C. Oncologia Medica, Azienda Ospedaliero Universitaria Sant'Andrea, Rome, Italy \\ *Author for correspondence: alain.gelibter@uniroma1.it
}

“'Recently, clinical evidence has raised BMI as an emerging prognostic factor for immunotherapy, regardless of cancer types"

First draft submitted: 20 December 2019; Accepted for publication: 10 February 2020; Published online: 25 February 2020

Keywords: $\mathrm{BMI} \bullet$ cancer $\bullet$ immune checkpoint inhibitors $\bullet$ immune system $\bullet$ immunotherapy $\bullet$ NSCLC $\bullet$ obesity $\bullet$ PD-1 • prognostic clinical marker

Immune checkpoint inhibitors (ICIs) have revolutionized systemic treatment of advanced not oncogene addicted NSCLC patients in the setting of first and latter lines. However, despite improvements in overall survival (OS) and clinical responses, most patients with NSCLC fail to respond to PD-1/PD-L1 axis inhibitors [1-5]. Clinical and molecular markers that could effectively predict the efficacy of anti PD-1/PD-L1 are crucial for NSCLC patient selection in clinical practice. The most established marker is the PD-L1 expression status on the membrane of tumor and immune cells, which is associated with enhanced response rates to PD-1/PD-L1 inhibition, but it has a limited sensitivity and specificity $[1,2,5-7]$. Recently, clinical evidence has raised BMI as an emerging prognostic factor for immunotherapy, regardless of cancer types [8-11].

In a pooled analysis of 4090 cancer patients under ICIs treatment, $\mathrm{Xu}$ and colleagues investigated the correlation between BMI and patient survival, regardless of cancer types and treatment setting [12]. Progression free survival (PFS), OS and response were the main outcome indicators. Among the 16 trials evaluated, the authors found a statistically significant difference in reduction of risk of mortality between patients with high BMI and patients with low BMI. Moreover, PFS and objective response rate in cancer patients with high BMI were improved, and the prognostic role of BMI was independent of sex.

An important article regarding inflammatory pathways, BMI and renal cancer has been recently published [13]. Sanchez and colleagues investigated the tumor immunological profile of patients with clear cell renal carcinoma, according to BMI value and categories. In particular, they investigated the transcriptomic profiles of tumors by an angiogenic and immunological point of view, and they related the results to perinephric adipose tissue in patients with a normal weight (i.e., with a BMI $18.5-24.9 \mathrm{~kg} / \mathrm{m}^{2}$, as per WHO's BMI categories) and in obese patients $\left(B M I \geq 30 \mathrm{~kg} / \mathrm{m}^{2}\right)$ in five cohorts of patients with clear cell renal carcinoma. In their analysis, they found that tumors arising in obese people inactivated interferon and other inflammatory pathways, while they overexpressed some pro-angiogenetic signals like transforming TGF- $\beta$, hypoxia inducible factors, VEGF and epithelial-mesenchymal transitions. In addition, the tumors of overweight patients showed a higher concentration of plasmacytoid dendritic cells and a lower proportion of innate lymphoid cells, that could be involved in cancer response to therapies, including ICIs. Despite the potential increase risk of developing cancer in obese people, these results suggested that BMI could be used as a prognostic marker for cancer patients treated with ICIs and could improve clinical outcomes. But is this true for overweight NSCLC patients too? 
In Xu's analysis, it was reported the subtotal risk of death for two studies that considered only NSCLC patients (hazard ratio $[\mathrm{HR}]=0.82$ ) and the global HR for PFS from eight NSCLC studies (HR: 0.75; $\mathrm{p}=0.09$ ) [12]. All available data are retrospective and prospective studies are warranted to validate these findings.

Recently, we conducted a 'real-life', multicenter, retrospective observational study that aimed to compare the clinical outcomes of cancer patients treated with ICIs according to baseline BMI levels. We enrolled 976 advanced cancer patients treated with anti-PD-1/PD-L1 immunotherapy, and we discovered a significant association between overweight patients (BMI $\geq 25)$ and improved clinical outcomes to ICIs [14].

We found that median time to treatment failure was significantly longer in overweight/obese patients compared with non-overweight patients (9.3 [95\% CI: 8.1-11.6; 318 events] versus 3.6 months [95\% CI: 3.2-4.1; 363 events]; HR: 0.51 [95\% CI: 0.44-0.60]; p < 0.0001). Similarly, median PFS was significantly improved in the overweight/obese group compared with the nonoverweight group (11.7 months [95\% CI: 9.4-15; 286 events] versus 3.7 months [95\% CI: 3.2-4.1; 358 events]; HR: 0.46 [95\% CI: 0.39-0.54]; p < 0.0001). We also consistently observed a significantly prolonged median OS among overweight/obese patients compared with non-overweight patients (26.6 months [95\% CI: 21.4-36.8; 286 censored patients] versus 6.6 months [95\% CI: 5.8-8.5; 182 censored patients]; HR: 0.33 [95\% CI: 0.28-0.41]; p < 0.0001).

In a recent pooled analysis of the International Lung Cancer Consortium (Lyon, France), Shepshelovich $e t$ al. identified a relationship between BMI at diagnosis and OS in patients with NSCLC, with the worst prognosis in underweight and morbidly obese patients [15]. Smoking, sex and ethnicity heterogeneity were reported in the prognostic relationship, with BMI at diagnosis, in their study.

The association between BMI and response to immunotherapy arise from different evidences in preclinical setting and a strong biological rational. In fact, obesity is basically associated with an immunosuppressive phenotype and proinflammatory status with a decreasing in M2 macrophages, CD8 T cells and NK T cells. In addition, preclinical studies showed that T-cell exhaustion in tumor-bearing mice is partly ruled by leptin signaling. This upregulates PD1 level expression and affects antitumor immunity leaving tumors more responsive to checkpoint blockade [16]. The leptin-induced activation of the JAK/STAT pathway in overweight patients could also lead to PD-L1 upregulation on tumor cells by IFN- $\gamma$.

We believe that obesity should not be considered as a positive prognostic factor but rather as a mediator of immune dysfunction and tumor progression that can be successfully reversed by PD-1/PD-L1 inhibition. Correlation of the favorable role of increased BMI and PD-L1 expression status should be prospectively well indagated in the future. This could be a crucial issue in advanced NSCLC where PD-L1 status is the only available biomarker and at least not conclusive for immune checkpoint inhibition treatment choice.

\section{Financial \& competing interests disclosure}

The authors have no relevant affiliations or financial involvement with any organization or entity with a financial interest in or financial conflict with the subject matter or materials discussed in the manuscript. This includes employment, consultancies, honoraria, stock ownership or options, expert testimony, grants or patents received or pending, or royalties.

No writing assistance was utilized in the production of this manuscript.

\section{Open access}

This work is licensed under the Attribution-NonCommercial-NoDerivatives 4.0 Unported License. To view a copy of this license, visit http://creativecommons.org/licenses/by-nc-nd/4.0/

\section{References}

1. Herbst RS, Baas P, Kim DW et al. Pembrolizumab versus docetaxel for previously treated, PD-L1-positive, advanced non-small-cell lung cancer (KEYNOTE-010): a randomised controlled trial. Lancet 387(10027), 1540-1550 (2016).

2. Borghaei H, Paz-Ares L, Horn L et al. Nivolumab versus docetaxel in advanced nonsquamous non-small-cell lung cancer. N. Eng. J. Med. 73(17), 1627-1639 (2015).

3. Brahmer J, Reckamp KL, Baas P et al. Nivolumab versus docetaxel in advanced squamous-cell non-small-cell lung cancer. N. Eng. J. Med. 373(2), 123-135 (2015).

4. Rittmeyer A, Barlesi F, Waterkampp D et al. Atezolizumab versus docetaxel in patients with previously treated non-small-cell lung cancer (OAK): a phase 3, open-label, multicentre randomised controlled trial. Lancet 389(10066), 255-265 (2017).

5. Topalian SL, Taube JM, Anders RA, Pardoll DM. Mechanism-driven biomarkers to guide immune checkpoint blockade in cancer therapy. Nat. Rev. Cancer 16(5), 275-287 (2016). 
6. Tumeh PC, Harview CL, Yearley JH et al. PD-1 blockade induces responses by inhibiting adaptive immune resistance. Nature 515(7528), 568-571 (2014).

7. Herbst RS, Soria JC, Kowanetz M et al. Predictive correlates of response to the anti-PD-L1 antibody MPDL3280A in cancer patients. Nature 515(7528), 563-567 (2014).

8. Murphy WJ, Longo DL. The surprisingly positive association between obesity and cancer immunotherapy efficacy. JAMA 321(13), 1247-1248 (2019).

9. Fang S, Wang Y, Dang Y et al. Association between body mass index, C-reactive protein levels, and melanoma patient outcomes. J. Invest. Dermatol. 137(8), 1792-1795 (2019).

10. Rassy EE, Ghosn M, Rassy NA, Assi T, Robert C. Do immune checkpoint inhibitors perform identically in patients with weight extremes? Immunotherapy 10(9), 733-736 (2018).

11. McQuade JL, Daniel CR, Hess KR et al. Association of body-mass index and outcomes in patients with metastatic melanoma treated with targeted therapy, immunotherapy, or chemotherapy: a retrospective, multicohort analysis. Lancet Oncol. 19(3), 310-322 (2018).

12. $\mathrm{Xu} \mathrm{H}, \mathrm{Cao} \mathrm{D}, \mathrm{He} \mathrm{A}, \mathrm{Ge} \mathrm{W}$. The prognostic role of obesity is independent of sex in cancer patients treated with immune checkpoint inhibitors: a pooled analysis of 4090 cancer patients. Int. Immunopharmacol. 74, 105745 (2019).

13. Sanchez A, Furberg H, Kuo F et al. Transcriptomic signatures related to obesity paradox in patients with clear renal cell carcinoma: a cohort study. Lancet Oncol. 21(2), 283-293 (2019).

14. Cortellini A, Bersanelli M, Buti S et al. A multicenter study of body mass index in cancer patients treated with anti-PD-1/PD-L1 immune checkpoint inhibitors: when overweight becomes favorable. J. Immunother. Cancer 7(1), 57 (2019).

15. Shepshelovich D, Xu W, Lu L et al. Body mass index (BMI), BMI change, and overall survival in patients with SCLC and NSCLC: a pooled analysis of the International Lung Cancer Consortium. J. Thorac. Oncol. 14(9), 1594-1607 (2019).

16. Wang Z, Aguilar EG, Luna JI et al. Paradoxical effects of obesity on T cell function during tumor progression and PD-1 checkpoint blockade. Nat. Med. 25(1), 141-151 (2018). 
